\title{
A lateral flow assay for the rapid diagnosis of Mycobacterium bovis infection in wild boar
}

\author{
Alba Fresco-Taboada ${ }^{1}$ (D) | María A. Risalde ${ }^{2,3,4}$ (D) | Christian Gortázar ${ }^{2}$ (D) | Istar Tapia ${ }^{1}$ | \\ Isabel González ${ }^{1}$ | Ángel Venteo ${ }^{1}$ (D) | Antonio Sanz ${ }^{1}$ | Paloma Rueda ${ }^{1}$ (D)
}

${ }^{1}$ Inmunología y Genética Aplicada, S.A. (INGENASA), Madrid, Spain

${ }^{2}$ SaBio (Health and Biotechnology), Instituto de Investigación en Recursos Cinegéticos IREC (CSIC-UCLM), Ciudad Real, Spain

${ }^{3}$ Dpto. de Anatomía y Anatomía

Patológica Comparadas, Facultad de Veterinaria, Universidad de Córdoba (UCO), Agrifood Excellence International Campus (ceiA3), Córdoba, Spain

${ }^{4}$ Infectious Diseases Unit, Hospital Universitario Reina Sofía de Córdoba, Instituto Maimonides de Investigación Biomédica de Córdoba (IMIBIC), University of Córdoba, Córdoba, Spain

\section{Correspondence}

Paloma Rueda, Inmunología y Genética Aplicada, S.A. (INGENASA), Madrid, Spain. Email: prueda@ingenasa.com

Funding information

Seventh Research Framework Program FP7-KBBE-2013-7, Grant/Award Number: 613799; MINECO and EU-FEDER, Grant/ Award Number: CGL2017-89866

\begin{abstract}
The native Eurasian wild boar (Sus scrofa) is a reservoir of Mycobacterium bovis, the causative agent of animal tuberculosis (TB), a chronic disease in livestock, companion animals and wild mammals. Cases of M. bovis infection in wild boar or feral pig have been reported worldwide, making early detection a priority in the eradication of the disease. Point-of-care diagnostic tests, such as low cost lateral flow assays, provide high specificity and sensitivity and can be performed on site, an essential requirement for a rapid screening of wildlife. A lateral flow assay, LFA, (INgezim TB CROM Ab) for the detection of M. bovis-specific antibodies in wild boar serum and blood has been developed based on MPB83, one of the major immunogenic antigens of the bacterium. A total of 140 samples of wild boar serum, well-characterized by Mycobacterium tuberculosis complex culture and TB compatible post-mortem lesions, have been analysed with LFA, and results were compared with one in-house and two commercial Enzyme-linked Immunosorbent Assays (ELISA), INgezim TB Porcine and INgezim Tuberculosis DR. In experimental samples, the achieved values of sensitivity of the different techniques ranged from $84.3 \%$ to $92.1 \%$ and the specificity was $100 \%$ in all of them. In field animals, specificity ranged from $96 \%$ to $100 \%$, whereas sensitivity ranged from $48 \%$ to $64 \%$ in juvenile wild boar, increasing to $93.3 \%-100 \%$ in adult wild boar. In particular, the total sensitivity and specificity values obtained with the new LFA were $83 \%$ and $97 \%$, respectively, indicating that INgezim TB CROM Ab could be used as a first approach for the surveillance of TB in wild boar, with a special applicability for animal-side testing.
\end{abstract}

KEYWORDS

enzyme-linked immunosorbent assay, lateral flow assay, tuberculosis, wild boar

\section{1 | INTRODUCTION}

Animal tuberculosis (TB) caused by Mycobacterium bovis is a chronic and important disease that affects both domestic and wild mammals and can spill over to human beings. The native Eurasian wild boar (Sus scrofa) is a reservoir of $M$. bovis, especially in the Iberian Peninsula (Gortázar, Che Amat, \& O'Brien, 2015; Naranjo, Gortázar, Vicente, \& Fuente, 2008). Although reporting the presence of TB in wild boar is not mandatory, cases of $M$. bovis infection in wild boar (or feral pigs and their crossbreds) have been detected worldwide, sometimes linked to outbreaks in cattle (Gortázar et al., 2012; Richomme et al., 2013). Thus, active surveillance of wild boar is taking place in several European countries (Riviere, Carabin, Strat, Hendrikx, \& Dufour, 2014) and in the USA (Pedersen et al., 2017).

Detection of TB has been traditionally based on cell-mediated immunity, although humoral immunity has gained ground in the last 
years, especially in swine, where humoral response to MTC is very remarkable (Thomas et al., 2019). Several serological tests such as Enzyme-Linked Immunosorbent Assays (ELISA) or Lateral Flow Assays (LFA) have recently been exploited for the detection of the humoral response to $M$. bovis infection in different species (Bezos et al., 2014; Casal et al., 2017; Infantes-Lorenzo et al., 2019; Thomas et al., 2019). LFAs have been described to analyse elephant, cervid and non-human primate sera, with sensitivities ranging from $79 \%$ in elk to $100 \%$ in elephants (Lyashchenko, Gortazar, Miller, \& Waters, 2018; Lyashchenko et al., 2007). This LFA has also been tested in wild boar serum, providing high specificity and sensitivity (Che' Amat et al., 2015), but with the limitation that it only detects IgGs (Boadella et al., 2011).

Here, we report the development of a new rapid test for the detection of $M$. bovis-specific antibodies, as a tool for the rapid screening of animal TB in comparison with one in-house and two commercial ELISAs (INgezim TB Porcine and INgezim Tuberculosis $\mathrm{DR})$.

\section{2 | MATERIALS AND METHODS}

\section{1 | Study area and sampling}

This study was carried out with a total of 140 experimental and field serum samples of wild boar (Sus scrofa).

Experimental samples: Nine sera from non-infected wild boar and 51 sera from infected wild boar.

A high challenge dose of $10^{6} \mathrm{cfu}$ of M. bovis by the oropharyngeal route was used in order to obtain uniform results, as was described in several experiments (Ballesteros et al., 2009; Beltrán-Beck et al., 2014; Garrido et al., 2011, 2010). As a consequence, all wild boar subjected to experimental challenge became infected. However, this challenge protocol may have led us to overestimate the antibodies detection, which might be lower under field conditions with natural exposure to lower challenge doses. Blood samples were taken at 2 , $4,6,10,16,18$ and 42 weeks after challenge with M. bovis.

Field samples were classified based on the presence or absence of TB compatible lesions (TBL) and MTC (Mycobacterium tuberculosis complex) culture isolation: 25 TB-positive juveniles (animals <12 months old based on tooth eruption patterns; Saenz de Buruaga, Lucio, \& Purroy, 1991) and 55 adults of more than 12 months old (30 sera from TB-positive wild boar and 25 sera from TB-negative wild boar). A TB-positive animal is defined as the one that has TBL and is also positive to the MTC culture, while a TB-negative animal is negative with regard to both TBL and the MTC culture.

All the animals were subjected to a detailed post-mortem examination in order to assess the presence or absence of TB compatible lesions (TBL). Tissues with present or suspicious TBLs were cultured for MTC. When TBLs were not observed, a pool of mandibular, tracheobronchial, and mediastinal lymph nodes (LNs) from each animal was also cultured.

These samples were placed in a sterile recipient and stored at $-80^{\circ} \mathrm{C}$ until its bacteriological culture. Culture was performed on Coletsos and $0.2 \%(\mathrm{w} / \mathrm{v})$ pyruvate-enriched Löwenstein-Jensen media (BD Diagnostics - Diagnostic Systems) after decontamination with a final concentration of $0.37 \%$ hexadecylpyridinium chloride. Isolates were identified by PCR and spoligotyping, as was described previously (Thomas et al., 2019).

\section{2 | Preparation of lateral flow strips and test procedure}

The qualitative assay was a direct lateral flow assay in which both the detector and capture reagent were based on the MPB83 purified recombinant protein (Figure 1). To produce the capture reagent, MPB83 and a monoclonal antibody specific for a control protein (BSA-biotin) were dispensed in two parallel lines respectively on a nitrocellulose membrane (Millipore) using a BIODOT Dispensing Platform XYZ 3,060. The detector reagent consisted of $300 \mathrm{~nm}$ red carboxyl-modified latex microspheres (IKERLAT polymers) covalently coated with the recombinant MPB83 purified protein (rMPB83), and green nanoparticles coated with BSA-biotin. The mixture of latex beads was dispensed onto the conjugate pad (Alhstrom) using a MATRIX 1600 Dispensing Platform.

Finally, the nitrocellulose membrane, conjugate pad, absorbent pad and sample pad (Cytosep 1662, Alhstrom) were assembled and then cut to $7.2 \mathrm{~mm}$ width strips that were placed in a plastic house.

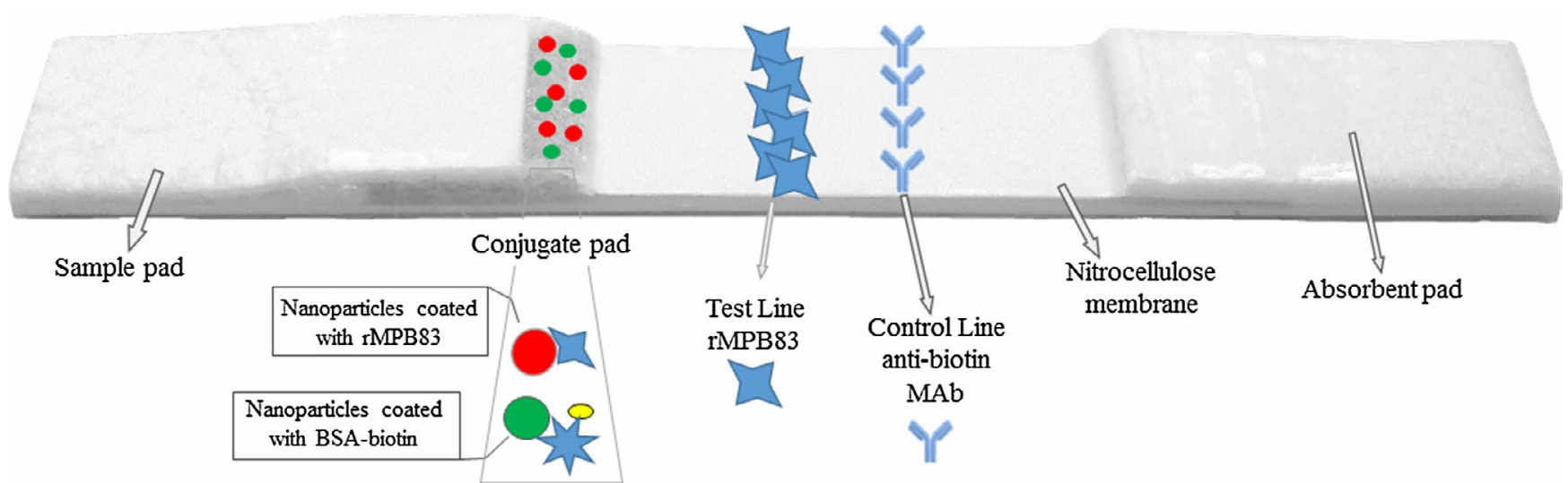

FIGURE 1 Diagram of the MPB83-based lateral flow assay for the detection of Mycobacterium bovis-specific antibodies 
TAB LE 1 Experimental samples from non-infected and Mycobacterium bovis-infected wild boar: Estimates of sensitivity and specificity with $95 \%$ confidence intervals $(95 \% \mathrm{Cl})$ and concordance values for each diagnostic test with TB-status $(n=60)$

\begin{tabular}{|c|c|c|c|c|c|c|c|}
\hline Diagnostic test & $\begin{array}{l}\text { Number of positive } \\
\text { samples }\end{array}$ & \multicolumn{2}{|c|}{ Sensitivity } & $\begin{array}{l}\text { Number of negative } \\
\text { samples }\end{array}$ & \multicolumn{2}{|c|}{ Specificity } & $\begin{array}{l}\text { Concordance } \\
\kappa\end{array}$ \\
\hline $\begin{array}{l}\text { INgezim TB CROM } \\
\mathrm{Ab}\end{array}$ & $46 / 51$ & 90.2 & $78.6-96.7$ & $9 / 9$ & 100 & $66.2-100$ & 0.69 \\
\hline INgezim TB Porcine & $47 / 51$ & 92.1 & 81.1-97.8 & $9 / 9$ & 100 & $66.2-100$ & 0.71 \\
\hline $\begin{array}{l}\text { INgezim } \\
\text { Tuberculosis DR }\end{array}$ & $44 / 51$ & 86.2 & $73.7-94.3$ & $9 / 9$ & 100 & $66.2-100$ & 0.69 \\
\hline In-house ELISA & $43 / 51$ & 84.3 & $71.4-93.0$ & $9 / 9$ & 100 & $66.2-100$ & 0.62 \\
\hline
\end{tabular}

The test procedure was carried out as follows: serum samples, obtained after centrifuging the blood for $10 \mathrm{~min}$, were $\mathbf{5 0}$-fold diluted in running buffer, and a volume of $150 \mu$ was added drop by drop to the round window of the LFA. Results were read $12 \mathrm{~min}$ after the addition of the diluted sample. The whole process could last up to 25-30 min. The appearance of two lines indicates a positive result, being the appearance of the upper line (Control line) mandatory to consider the test valid.

\subsection{ELISA tests}

Performance of the INgezim TB CROM Ab was compared to the performance of two commercial ELISA tests, INgezim Tuberculosis DR (based on MPB83) and INgezim TB Porcine (based on MPB83 and MPB70), employed following the manufacturer's instructions; and one in-house indirect ELISA (based on P22 protein complex; Thomas et al., 2019).

\subsection{Data analysis}

Sensitivity (Se), Specificity (Sp), 95\% confidence intervals (95\% Cl) and Kappa values $(\kappa)$ were assessed using the MedCalc for Windows, version 10.1.7.0 (MedCalc Software).

\section{3 | RESULTS AND DISCUSSION}

A panel of 60 serum samples from experimental wild boar and 80 field serum samples (55 TB-positive and 25 TB-negative) were analysed by
LFA and by the three ELISA tests used for comparison in this study. When samples from experimentally infected animals were assayed, 46 out of the 51 sera from infected wild boar were classified as TB-positive by the lateral flow test (INgezim TB CROM Ab). The comparison between the performance of the LFA and the ELISA tests showed that only the sensitivity of one commercial ELISA was slightly higher than the one obtained by LFA (Table 1). Regarding specificity, all TB-negative samples were classified as negative with the INgezim TB CROM Ab as well as with the three reference ELISAs (in-house, Tuberculosis DR and TB Porcine).

Concerning field serum samples, wild boar were divided into juveniles and adults, based on tooth eruption patterns. In adults, only 2 out of 30 TB-positive sera were not detected using the INgezim TB CROM $\mathrm{Ab}$, and one TB-negative serum was detected as false positive. The sensitivity and specificity values shown in Table 2 were higher than the ones obtained when the same test was employed for the detection of antibodies in a panel of 129 free-range pigs (Cardoso-Toset et al., 2017).

However, when 25 juvenile wild boar classified as positive by TBL and MTC culture were assayed, the sensitivity achieved by the INgezim TB CROM Ab was 56\%, $64 \%$ by the INgezim TB Porcine and $48 \%$ by the INgezim Tuberculosis DR (Table 3 ). Surprisingly, the sensitivity obtained with the in-house P22 ELISA was 96\%, indicating that the performance of this assay is independent of the age of the wild boar, as Boadella et al. (2011) described for an ELISA based on bPPD (bovine Protein Purified Derivative). P22 is a complex of proteins composed of several antigens including MPB70, MPB83, ESAT-6 and CFP-10 (Infantes-Lorenzo et al., 2017). Some juvenile wild boar could have produced antibodies against any protein contained in the

TAB LE 2 Adult field samples from non-infected and Mycobacterium bovis-infected wild boar: Estimates of sensitivity and specificity with $95 \%$ confidence intervals $(95 \% \mathrm{Cl})$ and concordance values for each diagnostic test with bacterial culture $(n=55)$

\begin{tabular}{|c|c|c|c|c|c|c|c|}
\hline Diagnostic test & $\begin{array}{l}\text { Number of } \\
\text { positive samples }\end{array}$ & \multicolumn{2}{|c|}{ Sensitivity } & $\begin{array}{l}\text { Number of } \\
\text { negative samples }\end{array}$ & \multicolumn{2}{|c|}{ Specificity } & $\begin{array}{l}\text { Concordance } \\
\kappa\end{array}$ \\
\hline INgezim TB CROM Ab & $28 / 30$ & 93.3 & $77.9-99.0$ & $24 / 25$ & 96.0 & $79.6-99.3$ & 0.89 \\
\hline INgezim TB Porcine & $30 / 30$ & 100 & $88.3-100$ & $25 / 25$ & 100 & $86.2-100$ & 1 \\
\hline $\begin{array}{l}\text { INgezim Tuberculosis } \\
\text { DR }\end{array}$ & $28 / 30$ & 93.3 & $77.9-99.0$ & $25 / 25$ & 100 & $86.2-100$ & 0.93 \\
\hline
\end{tabular}




\begin{tabular}{|c|c|c|c|c|}
\hline \multirow[b]{2}{*}{ Diagnostic test } & \multirow{2}{*}{$\begin{array}{l}\text { Number of } \\
\text { positive samples }\end{array}$} & \multicolumn{2}{|c|}{ Sensitivity } & \multirow{2}{*}{$\begin{array}{l}\text { Concordanc } \\
\kappa\end{array}$} \\
\hline & & $\%$ & $95 \% \mathrm{Cl}$ & \\
\hline INgezim TB CROM Ab & $14 / 25$ & 56 & $34.9-75.6$ & 0.437 \\
\hline INgezim TB Porcine & $16 / 25$ & 64 & $42.5-82.0$ & 0.521 \\
\hline INgezim Tuberculosis DR & $12 / 25$ & 48 & $27.8-68.7$ & 0.321 \\
\hline In-house ELISA & $24 / 25$ & 96 & 76.7-99.9 & 0.936 \\
\hline
\end{tabular}

TAB LE 3 Juvenile Mycobacterium bovis-infected wild boar field samples: Estimates of sensitivity with $95 \%$ confidence intervals $(95 \% \mathrm{Cl})$ and concordance values for each diagnostic test with mycobacterial culture $(n=25)$

TAB LE 4 Kappa values $(\kappa)$ and $p$ value between the evaluated serologic assays

\begin{tabular}{|c|c|c|c|c|}
\hline Diagnostic test & $\begin{array}{l}\text { INgezim TB } \\
\text { CROM Ab }\end{array}$ & $\begin{array}{l}\text { INgezim TB } \\
\text { Porcine }\end{array}$ & $\begin{array}{l}\text { INgezim } \\
\text { Tuberculosis DR }\end{array}$ & In-house ELISA \\
\hline $\begin{array}{l}\text { INgezim TB CROM } \\
\mathrm{Ab}\end{array}$ & 1.0 & & & \\
\hline INgezim TB Porcine & 0.781 & 1.0 & & \\
\hline $\begin{array}{l}\text { INgezim Tuberculosis } \\
\text { DR }\end{array}$ & 0.819 & 0.816 & 1.0 & \\
\hline In-house P22 ELISA & 0.641 & 0.711 & 0.625 & 1.0 \\
\hline
\end{tabular}

Note: $\kappa \leq 0.20$ : none to slight agreement; $\kappa=0.21-0.40$ : fair agreement; $\kappa=0.41-0.60$ : moderate agreement; $\kappa=0.61-0.80$ : substantial agreement; $\kappa \geq 0.81$ : almost perfect agreement. $p$ value for all cases was $<0.0001$ (obtained applying Chi-square test).

P22 complex apart from MPB83 and MPB70, explaining why some samples are not recognized by the INgezim tests. Negative samples of this group of juvenile animals should be assayed. Furthermore, the difference of sensitivity found among animals of different ages when INgezim tests were used could be explained due to the fact that in adult wild boar, antibody production is closely related to infection status/lesion severity, whereas lesions can appear in juvenile wild boar exposed to a great burden of M. bovis, but, due to their younger age, the production of MPB83/70 specific antibodies has not reached a detectable level yet; as already shown by Che' Amat et al., 2015.

Regarding concordance between assays (Table 4), agreement was substantial in all cases, but it was almost perfect between the LFA and INgezim Tuberculosis DR. This result was expected since the format of the two assays is equivalent, a double recognition of the antigen by the antibodies present on the sample based on the protein MPB83.

In conclusion, these results show that the newly developed lateral flow assay, INgezim TB CROM Ab, is a suitable tool for the detection of M. bovis-specific antibodies and that it could be used as a reliable first assay for the surveillance of TB in adult wild boar. In juveniles, lateral flow tests have a lower sensitivity and parallel testing with ELISA assays is recommended.

\section{ACKNOWLEDGEMENTS}

Part of this research was funded by the EU, Seventh Research Framework Program FP7-KBBE-2013-7 under grant number $\mathrm{n}^{\circ}$ 613799 (WildTBVac). This is a contribution to the WildDriver grant CGL2017-89866 from MINECO and EU-FEDER. We are very grateful to Dr. Mercedes Dominguez and her laboratory (Instituto de Salud Carlos III, Unidad de Inmunología Microbiana, Majadahonda, Spain) for their generous supply of P22 protein complex. We also thank Neiker and Visavet for providing valuable samples.

\section{CONFLICT OF INTEREST}

A. Fresco, I. Tapia, I. González, A. Venteo, A. Sanz and P. Rueda are employees of INGENASA, the manufacturing company of the INgezim LFA and ELISA tests. M.A. Risalde and C. Gortázar declare no conflict of interest.

\section{ETHICAL STATEMENT}

In the experimental animals, the handling and sampling frequency procedures were designed to reduce stress and health risks for the subjects, according to European (86/609) and Spanish legislation (R.D. 223/1988 and R.D. 1,021/2005). The protocol was approved by the Committee on the Ethics of Animal Experiments of the Regional Agriculture Authority (Diputación Foral de Vizcaya, Permit Number: 2,731-2009; Comunidad de Madrid, permit CM180112-01). The field animals were not intentionally killed for this study but were hunted legally during the hunting season with appropriate permits. No ethical approval was, therefore, deemed necessary for this study. Protocols, amendments and other resources were used according to the guidelines approved by each autonomous government following the R.D.1201/2005 of the Spanish Ministry of Presidency. Blood and tissue samples were collected by veterinarians in compliance with the Ethical Principles in Animal Research.

\section{ORCID}

Alba Fresco-Taboada (iD https://orcid.org/0000-0001-7103-8522

María A. Risalde (iD https://orcid.org/0000-0001-6751-1305

Christian Gortázar (iD https://orcid.org/0000-0003-0012-4006

Ángel Venteo (iD https://orcid.org/0000-0002-3680-5906

Paloma Rueda iD https://orcid.org/0000-0002-7735-3766 


\section{REFERENCES}

Ballesteros, C., Garrido, J. M., Vicente, J., Romero, B., Galindo, R. C., Minguijón, E., ... Gortázar, C. (2009). First data on Eurasian wild boar response to oral immunization with BCG and challenge with a Mycobacterium bovis field strain. Vaccine, 27, 6662-6668. https://doi. org/10.1016/j.vaccine.2009.08.095

Beltrán-Beck, B., de la Fuente, J., Garrido, J. M., Aranaz, A., Sevilla, I., Villar, M., ... Gortazar, C. (2014). Oral vaccination with heat inactivated Mycobacterium bovis activates the complement system to protect against tuberculosis. PLoS ONE, 9, e98048. https://doi. org/10.1371/journal.pone.0098048

Bezos, J., Casal, C., Romero, B., Schroeder, B., Hardegger, R., Raeber, A. J., ... Dominguez, L. (2014). Current ante-mortem techniques for diagnosis of bovine tuberculosis. Research in Veterinary Science, 97, S44-52. https://doi.org/10.1016/j.rvsc.2014.04.002

Boadella, M., Lyashchenko, K., Greenwald, R., Esfandiari, J., Jaroso, R., Carta, T., ... Gortázar, C. (2011). Serologic tests for detecting antibodies against Mycobacterium bovis and Mycobacterium avium subspecies paratuberculosis in Eurasian wild boar (Sus scrofa scrofa). Journal of Veterinary Diagnostic Investigation, 23, 77-83. https://doi. org/10.1177/104063871102300111

Cardoso-Toset, F., Luque, I., Carrasco, L., Jurado-Martos, F., Risalde, M. A., Venteo, A., ... Gomez-Laguna, J. (2017). Evaluation of five serologic assays for bovine tuberculosis surveillance in domestic freerange pigs from southern Spain. Preventive Veterinary Medicine, 137, 101-104. https://doi.org/10.1016/j.prevetmed.2016.12.016

Casal, C., Infantes, J. A., Risalde, M. A., Diez-Guerrier, A., Dominguez, M., Moreno, I., ... Bezos, J. (2017). Antibody detection tests improve the sensitivity of tuberculosis diagnosis in cattle. Research in Veterinary Science, 112, 214-221. https://doi.org/10.1016/j.rvsc.2017.05.012

Che' Amat, A., González-Barrio, D., Ortiz, J. A., Díez-Delgado, I., Boadella, M., Barasona, J. A., ... Gortázar, C. (2015). Testing Eurasian wild boar piglets for serum antibodies against Mycobacterium bovis. Preventive Veterinary Medicine, 121, 93-98. https://doi.org/10.1016/j.prevetmed.2015.05.011

Garrido, J. M., Sevilla, I. A., Beltrán-Beck, B., Minguijón, E., Ballesteros, C., Galindo, R. C., ... Gortázar, C. (2011). Protection against tuberculosis in Eurasian wild boar vaccinated with heat-inactivated Mycobacterium bovis. PLoS ONE, 6, e24905. https://doi.org/10.1371/ journal.pone.0024905

Garrido, J. M., Vicente, J., Carrasco-García, R., Galindo, R. C., Minguijón, E., Ballesteros, C., ... Gortázar, C. (2010). Experimental infection of Eurasian wild boar with Mycobacterium avium subsp. avium. Veterinary Microbiology, 144, 240-245. https://doi.org/10.1016/j. vetmic.2009.12.041

Gortázar, C., Che Amat, A., \& O'Brien, D. J. (2015). Open questions and recent advances in the control of a multi-host infectious disease: Animal tuberculosis. Mammal Review, 45, 160-175. https://doi. org/10.1111/mam.12042

Gortázar, C., Delahay, R. J., McDonald, R. A., Boadella, M., Wilson, G. J., Gavier-Widen, D., \& Acevedo, P. (2012). The status of tuberculosis in European wild mammals. Mammal Review, 42, 193-206. https://doi. org/10.1111/j.1365-2907.2011.00191.x

Infantes-Lorenzo, J. A., Dave, D., Moreno, I., Anderson, P., Lesellier, S., Gormley, E., ... Salguero, F. J. (2019). New serological platform for detecting antibodies against Mycobacterium tuberculosis complex in European badgers. Veterinary Medicine and Science, 5, 61-69. https:// doi.org/10.1002/vms3.134

Infantes-Lorenzo, J. A., Moreno, I., Risalde, M. A., Roy, A., Villar, M., Romero, B., ... Domínguez, M. (2017). Proteomic characterisation of bovine and avian purified protein derivatives and identification of specific antigens for serodiagnosis of bovine tuberculosis. Clinical Proteomics, 14, 36. https://doi.org/10.1186/s12014-017-9171-z

Lyashchenko, K. P., Gortazar, C., Miller, M. A., \& Waters, W. R. (2018). Spectrum of antibody profiles in tuberculous elephants, cervids, and cattle. Veterinary Microbiology, 214, 89-92. https://doi.org/10.1016/j. vetmic.2017.12.013

Lyashchenko, K. P., Greenwald, R., Esfandiari, J., Greenwald, D., Nacy, C. A., Gibson, S., ... McCombs, C. (2007). PrimaTB STAT-PAK assay, a novel, rapid lateral-flow test for tuberculosis in nonhuman primates. Clinical Vaccine Immunology, 14, 1158-1164. https://doi.org/10.1128/ cvi.00230-07

Naranjo, V., Gortázar, C., Vicente, J., \& de La Fuente, J. (2008). Evidence of the role of European wild boar as a reservoir of Mycobacterium tuberculosis complex. Veterinary Microbiology, 127, 1-9. https://doi. org/10.1016/j.vetmic.2007.10.002

Pedersen, K., Miller, R. S., Anderson, T. D., Pabilonia, K. L., Lewis, J. R., Mihalco, R. L., ... Gidlewski, T. (2017). Limited antibody evidence of exposure to Mycobacterium bovis in feral swine (Sus scrofa) in the USA. Journal of Wildlife Diseases, 53, 30-36. https://doi. org/10.7589/2016-07-164

Richomme, C., Boadella, M., Courcoul, A., Durand, B., Drapeau, A., Corde, Y., ... Boschiroli, M. L. (2013). Exposure of wild boar to Mycobacterium tuberculosis complex in France since 2000 is consistent with the distribution of bovine tuberculosis outbreaks in cattle. PLOS ONE, 8, e77842. https://doi.org/10.1371/journ al.pone.0077842

Riviere, J., Carabin, K., Le Strat, Y., Hendrikx, P., \& Dufour, B. (2014). Bovine tuberculosis surveillance in cattle and free-ranging wildlife in EU Member States in 2013: A survey-based review. Veterinary Microbiology, 173, 323-331. https://doi.org/10.1016/j. vetmic.2014.08.013

Saenz de Buruaga, M., Lucio, A. J., \& Purroy, F. J. (1991). Reconocimiento de sexo y edad en especies cinegéticas. Ediciones Leonesas SA. León: Edilesa.

Thomas, J., Infantes-Lorenzo, J. A., Moreno, I., Cano-Terriza, D., de Juan, L., García-Bocanegra, I., ... Risalde, M. A. (2019). Validation of a new serological assay for the identification of Mycobacterium tuberculosis complex-specific antibodies in pigs and wild boar. Preventive Veterinary Medicine, 162, 11-17. https://doi.org/10.1016/j.preve tmed.2018.11.004

How to cite this article: Fresco-Taboada A, Risalde MA, Gortázar C, et al. A lateral flow assay for the rapid diagnosis of Mycobacterium bovis infection in wild boar. Transbound Emerg Dis. 2019;66:2175-2179. https://doi.org/10.1111/tbed.13260 